\title{
Controle de Formigas Lava-pés: Onde Encontro Informações?
}

\author{
Lívia Maria Barreto Pinto ${ }^{1 *}$, Elisa Furtado Fernandes ${ }^{1} \&$ Fábio Prezoto $^{1}$ \\ ${ }^{1}$ Universidade Federal de Juiz, de Fora, UFJF, Instituto de Ciências Biológicas, Departamento de Zoologia, Juiz de Fora, MG. \\ *E-mail para correspondência: liviamariabp@gmail.com
}

\begin{abstract}
RESUMO
As formigas lava-pés são facilmente encontradas em ambientes urbanos e, devido ao seu comportamento agressivo, ferroam suas vítimas, o que pode desencadear reações alérgicas graves ou até mesmo a morte do indivíduo. O controle dessas formigas ainda não é bem estabelecido, no entanto, é possível encontrar diversas alternativas de controle tanto na internet como na literatura. Sendo assim, o objetivo desse trabalho foi realizar um levantamento quantitativo acerca dos métodos de controle de lava-pés disponíveis nas diferentes mídias de divulgação. A pesquisa foi realizada nas plataformas do Google e YouTube para sites e vídeos, respectivamente, e Web of Science e Google Acadêmico para produções científicas, nos últimos 22 anos. Os termos utilizados na pesquisa foram "como controlar formigas lava-pés" em sites e vídeos e "fire ants, control, Brazil" para a busca bibliográfica. Os métodos sugeridos para o controle de formigas de fogo foram divididos em três categorias: caseiros, químicos e controle biológico. Um total de 38 publicações (18 sites, 12 vídeos e oito produções bibliográficas) que abordaram 18 métodos diferentes de controle foram registrados. Os métodos caseiros foram os mais citados $(n=39)$, seguido dos métodos químicos $(n=19)$ e controle biológico $(n=2)$. Dentre os sites encontrados, o uso de substâncias de origem vegetal $(n=7)$ foi o mais citado, já a maioria dos vídeos acessados sugeriram uma solução de vinagre com detergente $(n=5)$ e nas produções bibliográficas o uso de inseticidas $(n=4)$ foi mais recomendado. Houve participação de profissionais em $61 \%$ das publicações. Embora a internet seja um instrumento de pesquisa popular, é necessário atentar-se à confiabilidade das informações disponibilizadas, visto que o manuseio de determinados produtos pode ser nocivo à saúde e ao meio ambiente e em muitos casos ser totalmente ineficaz para o controle destas formigas.
\end{abstract}

Palavras-chave: Formigas de fogo, Métodos de controle, Pragas urbanas.

\section{ABSTRACT}

Control of the fire ants: where do I find information? The fire ants are easily found in urban environments, and because of their aggressive behavior, they sting their victims, which can trigger serious allergic reactions or even death. The control of these ants is still not well established, however, it is possible to find several control alternatives both in the internet and in the literature. Therefore, the objective of this work was to perform a quantitative survey on the methods of control of fire ants available in the different media of disclosure. The survey was conducted on Google and YouTube platforms for websites and videos, respectively, and Web of Science and Google Scholar for scientific productions, in the past 22 years. The terms used in the research were "how to control fire ants" in websites and videos and "fire ants, control, Brazil" for the bibliography search. The methods found were divided into three categories: homemade, chemical and biological control. A total of 38 publications (18 sites, 12 videos and eight bibliographic productions) that addressed 18 different methods of control were recorded. The homemade methods were the most cited $(n=39)$, followed by chemical methods $(n=19)$ and biological control $(n=2)$. Among the sites found, the use of substances of vegetable origin $(n=7)$ was the most cited, and most of the videos accessed suggested a solution of vinegar with detergent $(n=5)$ and in the bibliographical productions the use of insecticides $(n=4)$ was more recommended. There was participation of professionals in $61 \%$ of the publications. Although the internet is a popular research instrument, it is necessary to take into account the reliability of the information provided, since the handling of certain products can be harmful to health and the environment and in many cases be totally ineffective for the control of these ants.

Keywords: Control methods, Fire ants, Urban pests. 


\section{INTRODUÇÃO}

As ações humanas alteram significativamente o ambiente. Em áreas urbanizadas, por exemplo, o ambiente possui fatores abióticos próprios, que acabam por favorecer algumas espécies animais em detrimento de outras, especialmente pela grande concentração humana e disponibilidade de água e alimento (Botkin \& Keller, 2011). E dentre as várias populações de animais que habitam áreas modificadas pelo homem, como as áreas urbanas, estão centenas de espécies de formigas (Bueno \& Campos, 2017).

As formigas são insetos facilmente encontrados nos mais diferentes ambientes. Essa abundância faz com que algumas dessas espécies venham a ser consideradas pragas devido aos prejuízos econômicos que provocam, bem como pelo fato de afetarem direta e indiretamente o bem-estar humano e de animais (Campos-Farinha et al., 2002). Muitas dessas espécies causam apenas incômodo, enquanto outras ocupam equipamentos eletrônicos, ferroam ou são vetores de microrganismos patogênicos que podem afetar o homem e seus animais (Bueno \& Bueno, 2007; Magro, 2007).

Essa situação faz com que muitas pessoas busquem o controle destas formigas em suas casas, jardins ou cultivos por meio de informações disponíveis principalmente na internet. Contudo, nem sempre a recomendação encontrada nestas buscas foi comprovada cientificamente, gerando uma preocupação quanto ao seu emprego como estratégia de controle.

Dentre estas espécies podemos destacar as formigas lava-pés [Solenopsis (Westwood, 1840)], com especial atenção para a espécie Solenopsis invicta (Buren, 1972), que foi introduzida de forma não intencional no Sul dos Estados Unidos por meio do transporte marítimo (Creighton, 1930), no início do século XX. Mais recentemente esta espécie é responsável por milhares de acidentes com pessoas, além de enormes impactos ecológicos sobre as espécies nativas, bem como o gasto de milhões de dólares todos os anos devido aos prejuízos e o custo do controle (Cumberland \& Kirkman, 2012).

No Brasil, as espécies de lava-pés que mais se destacam são Solenopsis saevissima (Smith, 1855) e S. invicta, que devido à ampla distribuição territorial, causam impactos econômicos, prejuízos aos bens materiais, danos à saúde pública e ao bem-estar humano e de animais (Vinson \& Sorensen, 1986; Tschinkel, 2006; Zeringotá et al., 2014; Fernandes et al., 2016). Contudo, acredita-se que os prejuízos possam ser ainda maiores, uma vez que existem pouquíssimos estudos que enfoquem a ação destas formigas, seus danos, bem como estratégias de controle.

O controle de formigas lava-pés ainda não está estabelecido, no entanto, é possível encontrar facilmente diversas recomendações de como controlar estas formigas na internet. Dada a variedade de informações disponíveis, bem como a necessidade crescente de métodos de controle, o objetivo deste 
trabalho foi realizar um levantamento das informações disponíveis em sites, vídeos e produções bibliográficas do Brasil que indiquem métodos de controle de formigas lava-pés, visando assim analisar parâmetros destas informações, bem como a qualidade técnica de seu conteúdo.

\section{MATERIAL E MÉTODOS}

A pesquisa foi realizada em maio de 2019 , utilizando-se as plataformas Google e YouTube para busca de sites e vídeos, respectivamente, e as bases Web of Science e Google Acadêmico para produções bibliográficas no período de 1997 a 2019, no Brasil. As palavras-chave usadas nas pesquisas de sites e vídeos foram "como controlar formigas lava-pés", enquanto que, na busca de artigos científicos, foram "fire ants, control, Brazil".

Para cada publicação encontrada foram obtidas as seguintes informações: ano da publicação, tipo de publicação (produção bibliográfica, site ou vídeo), método de controle recomendado e se houve a participação de um especialista (pesquisadores e profissionais ligados ao controle de pragas) na publicação. Estas informações foram analisadas e apresentadas na forma de tabelas e gráficos ao longo do texto.

Os métodos de controle identificados foram categorizados em:

Caseiros (C): que envolvem a utilização de produtos de uso cotidiano, como artigos de limpeza (água sanitária, detergente) e substâncias de origem vegetal (casca de laranja, borra de café, óleos essenciais);

Químicos (Q): que abrangem pesticidas e iscas tóxicas;

Controle Biológico (CB): que indicam o uso de agentes biológicos (organismos patogênicos e parasitoides) para controlar as formigas lava-pés.

\section{RESULTADOS E DISCUSSÃO}

Ao todo foram localizadas 38 publicações (18 em sites, 12 em vídeos e oito produções bibliográficas), que recomendaram 18 diferentes métodos de controle de formigas lava-pés (Tabela 1). Cabe destacar que algumas publicações recomendam mais de um método de controle. Esses resultados demonstram que o material veiculado em sites e vídeos no YouTube são a maioria das informações disponíveis para controle (79\%) e, por isso, mais facilmente acessados por quem busca métodos de controle de formigas lava-pés do que as produções bibliográficas (11\%), o que revela a importância desses 
veículos como fontes de informação.

Na maioria dos casos, as publicações em sites e vídeos oferecem dois aspectos muito importantes para o visitante, que são: (1) o uso de uma linguagem mais acessível ao público leigo, o que facilita a compreensão e (2) riqueza de imagens, que favorece o reconhecimento das espécies pragas e o entendimento da metodologia de controle recomendada. Esses dois atributos, típicos destes meios de comunicação, os tornam diferenciados em relação às produções bibliográficas, que geralmente são apresentadas na forma de textos mais longos, com linguajar técnico e quase sempre sem imagens.

Dentre as categorias de controle recomendadas, os métodos caseiros foram os mais indicados (65\%; $n=39)$, seguidos pelos métodos químicos $(31,67 \% ; n=19)$ e controle biológico $(3,33 \% ; n=2)$ (Tabela 1).

Esses resultados corroboram o fato de que como ainda não foi estabelecido um controle efetivo para formigas lava-pés, estratégias alternativas acabam se popularizando nos meios de comunicação. $\mathrm{Na}$ maioria das publicações que recomendam métodos caseiros (vídeos e sites), a publicação valoriza o uso de substâncias de fácil acesso e métodos simples ao espectador como, por exemplo: água sanitária; água com detergente; água fervente; Lysoform ${ }^{\circledast}$; Creolina ${ }^{\circledR}$; casca de laranja; borra de café; carvão; bicarbonato; vinagre; talco e plantas (cravo-da-índia, alfazema, hortelã, pimenta, etc.) (Tabela 1).

Apesar da quantidade de publicações que recomendam métodos caseiros, cabe destacar que esses métodos nem sempre possuem eficiência comprovada pela ciência e por isso podem acarretar uma série de problemas como o risco durante sua preparação, no caso da água fervente, o risco durante a manipulação e aplicação como no caso do uso da água sanitária, Creolina ${ }^{\circledR}$, Lysoform ${ }^{\circledR}$, bicarbonato e vinagre, sem contar a ausência de informações sobre a quantidade/proporção a ser utilizada e os impactos gerados pelo emprego destes produtos no ambiente e para as espécies não-alvo (outros insetos, aves, animais domésticos, dentre outros). 
Tabela 1. Publicações sobre métodos de controle de formigas lava-pés nos últimos 22 anos no Brasil: autor, ano, meio de divulgação ( $P B=$ produções bibliográficas; $S=$ sites; $V=$ vídeos), descrição dos métodos e categorias de controle $(Q=$ químico; $\mathrm{C}=$ caseiro; $\mathrm{CB}=$ controle biológico). Os meios de divulgação com o símbolo "*” indicam a participação de especialistas (pesquisadores e profissionais da área).

\begin{tabular}{|c|c|c|c|c|}
\hline Fonte & Ano & Divulgação & Descrição do método & Controle \\
\hline Campos-Farinha et al. & 1997 & PB* & Água fervente, água sanitária diluída & $\mathrm{C}$ \\
\hline Campos-Farinha \& Bueno & 2004 & $\mathrm{~PB}^{*}$ & Água quente com detergente, inseticida & $\mathrm{C} / \mathrm{Q}$ \\
\hline Zorzenon \& Campos-Farinha & 2006 & $\mathrm{~PB}^{*}$ & Água sanitária diluída, inseticida & $C / Q$ \\
\hline Zuben et al. & 2006 & PB* & $\begin{array}{l}\text { Água com detergente, água sanitária diluída, iscas tóxi- } \\
\text { cas }\end{array}$ & $C / Q$ \\
\hline Bueno \& Bueno & 2007 & PB* & Limpeza local, água quente, iscas tóxicas, inseticidas & $\mathrm{C} / \mathrm{Q}$ \\
\hline Zarzuela & 2007 & PB* & Entomopatógenos & $\mathrm{CB}$ \\
\hline $\begin{array}{l}\text { www.jcnet.com.br/editorias_noticias.php?co- } \\
\text { digo=114775\&ano=2007\&p= }\end{array}$ & 2007 & $\mathrm{~S}^{*}$ & Água com detergente, iscas tóxicas & $\mathrm{C} / \mathrm{Q}$ \\
\hline $\begin{array}{l}\text { www.portalviva.com.br/index.php?op- } \\
\text { tion=com_content\&view=article\&id=350:conheca- } \\
\text { as-quatro-especies-de-formiga-que-infestam-sao- } \\
\text { paulo\&catid=7:sustentabilidade\&itemid=130 }\end{array}$ & 2009 & $S^{*}$ & $\begin{array}{l}\text { Limpeza do local, Cravo-da-índia, folha de louro ou tan- } \\
\text { gerina, água com detergente, iscas tóxicas }\end{array}$ & $\mathrm{C} / \mathrm{Q}$ \\
\hline www.youtube.com/watch?v=2ppw3wnijem & 2009 & $\mathrm{~V}^{*}$ & Remoção mecânica do formigueiro & c \\
\hline Souto et al. & 2011 & PB* & Biopesticida & Q \\
\hline $\begin{array}{l}\text { dedetizacaodepragas.com/dedetizacao-de-formigas } \\
\text { /dica-caseira-para-combater-as-formigas-lava-pe/ }\end{array}$ & 2012 & $S^{*}$ & Lysoform $^{\circledR}$ e Creolina ${ }^{\circledR}$ diluídos em água & C \\
\hline $\begin{array}{l}\text { www.f24.com.br/geral/casa/pragas/formigas/como- } \\
\text { eliminar-formigas }\end{array}$ & 2014 & Er & $\begin{array}{l}\text { Erva santa maria, talco, alfazema, álcool, borra do café, } \\
\text { etc. }\end{array}$ & C \\
\hline http://ruralnews.com.br/visualiza.php?id=816 & 2014 & $S^{*}$ & Formicida & Q \\
\hline $\begin{array}{l}\text { oextensionista.blogspot.com/2014/02/pratica-para- } \\
\text { o-manejo-ecologico-de.html\#.xnhrdy5kjiu }\end{array}$ & 2014 & $S^{*}$ & $\begin{array}{l}\text { Diversos métodos como farinha de osso, carvão vege- } \\
\text { tal, água fervendo, sapos, galinhas, etc. }\end{array}$ & $\mathrm{C} / \mathrm{CB}$ \\
\hline www.youtube.com/watch?v=oqwtzxwn5hc & 2014 & V & Água com vinagre branco e detergente & C \\
\hline www.youtube.com/watch?v=za5rofccyr4 & 2016 & V & Iscas tóxicas & Q \\
\hline www.youtube.com/watch?v=0uye4sfw0ty & 2016 & V & Água com vinagre branco e detergente & $\mathrm{C}$ \\
\hline www.youtube.com/watch?v=ttxqaeqpqmk & 2017 & $\mathrm{~V}$ & Óleos essenciais & C \\
\hline www.youtube.com/watch?v=27osaiisxli & 2017 & V & Água com detergente & C \\
\hline www.youtube.com/watch?v=ss7zmo1pnlc\&t=56s & 2017 & V & Borra de café & C \\
\hline $\begin{array}{l}\text { brcontrol.net/2018/09/26/grande-perigo-em-tama- } \\
\text { nho-pequeno-formiga-lava-pes-saiba-por-que-esta- } \\
\text { pequena-formiga-causa-serios-problemas/ }\end{array}$ & 2018 & $S^{*}$ & Dedetização & Q \\
\hline $\begin{array}{l}\text { ativaservicos.com/como-acabar-com-formigas-na- } \\
\text { sua-casa/ }\end{array}$ & 2018 & $S^{*}$ & $\begin{array}{l}\text { Sálvia, poejo, hortelã e menta (cheiro), spray de água, } \\
\text { sabão, iscas caseiras, vinagre, dedetização }\end{array}$ & $\mathrm{C} / \mathrm{Q}$ \\
\hline $\begin{array}{l}\text { melhorcomsaude.com.br/8-formas-eliminar-formi- } \\
\text { gas-maneira-organica/ }\end{array}$ & 2018 & $\mathrm{~S}$ & $\begin{array}{l}\text { Água de alho, arroz, cascas de laranja, levedura, pi- } \\
\text { menta branca, bicarbonato de sódio, infusão de ab- } \\
\text { sinto, limão e água }\end{array}$ & C \\
\hline www.youtube.com/watch?v=yongfyqzije & 2018 & V & $\begin{array}{l}\text { Mistura de água, vinagre de álcool, detergente, cravi- } \\
\text { nhos e naftalina ou cânfora }\end{array}$ & C \\
\hline www.youtube.com/watch?v=1ujbtsa2hyc & 2018 & V & Água com vinagre de maçã e detergente & $\mathrm{C}$ \\
\hline www.youtube.com/watch?v=z2kw8agdvx8 & 2018 & $\mathrm{~V}$ & Talco & $\mathrm{C}$ \\
\hline $\begin{array}{l}\text { revistagloborural.globo.com/vida-na-fazenda/gr-res- } \\
\text { ponde/noticia/2019/01/saiba-como-evitar-que-for- } \\
\text { migas-lava-pes-deixem-grama-seca.html }\end{array}$ & 2019 & $S^{*}$ & Bicarbonato de sódio com vinagre & C \\
\hline www.youtube.com/watch?v=wonfyfpcfzq & 2019 & $\mathrm{~V}$ & Fipronil diluído e água & Q \\
\hline www.youtube.com/watch?v=uwd53qpzugs & 2019 & V & Óleo queimado com detergente & $\mathrm{C}$ \\
\hline ra & 2012 & PB* & Pó de ostra & C \\
\hline $\begin{array}{l}\text { www.jardimdeflores.com.br/dicas/a19formigasejar- } \\
\text { dim.htm }\end{array}$ & - & $S^{*}$ & Água sanitária diluída, água fervente & $\mathrm{C}$ \\
\hline pt.wikihow.com/se-livrar-de-formigas-de-fogo & - & $S$ & Iscas, inseticidas, dedetização & Q \\
\hline $\begin{array}{l}\text { www.agrolink.com.br/problemas/formiga-lava- } \\
\text { pe_1875.html }\end{array}$ & - & $S$ & Inseticidas & Q \\
\hline $\begin{array}{l}\text { www.ddribeira.com.br/prevencao-de-formigas- } \\
\text { como-prevenir-infestacao-de-formigas/ }\end{array}$ & - & $\mathrm{S}^{*}$ & Água quente com detergente & $\mathrm{C}$ \\
\hline $\begin{array}{l}\text { pragas.com.br/consumidor/pragas3.php?id=234\&t } \\
\text { ppraga }=\mathrm{jdm}\end{array}$ & - & $\mathrm{S}^{*}$ & Água sanitária diluída, inseticidas & $\mathrm{C} / \mathrm{Q}$ \\
\hline $\begin{array}{l}\text { www.dedetizadoradesmatt.com.br/conteudo/con- } \\
\text { teudo.asp?id=25 }\end{array}$ & - & $S^{*}$ & Iscas, inseticidas & Q \\
\hline acaia.com.br/controle-de-pragas/ & - & $\mathrm{S}^{*}$ & Água sanitária diluída, inseticidas & $C / Q$ \\
\hline www.extermine.com $\cdot \mathrm{br} /$ ?pg=pragas-urbanas\&id=19 & - & $\mathrm{S}^{*}$ & Cravo-da-índia, folhas de louro, limão, tangerina, iscas & $\mathrm{C} / \mathrm{Q}$ \\
\hline
\end{tabular}


Em um experimento realizado em campo, testamos duas recomendações de controle caseiro para lava-pés, (a) o uso de limão com cravo da Índia e (b) borra de café. Como pode ser observado na Figura 1, é possível verificar as formigas se deslocando e atacando o limão com cravo (Figura 1A) e, no caso da borra de café, além de caminharem facilmente sobre o produto (Figura 1B), após o período de perturbação as formigas incorporaram a borra de café à estrutura do ninho durante a tarefa de reconstrução, demonstrando que não havia qualquer efeito negativo da presença dessa substância no ninho.
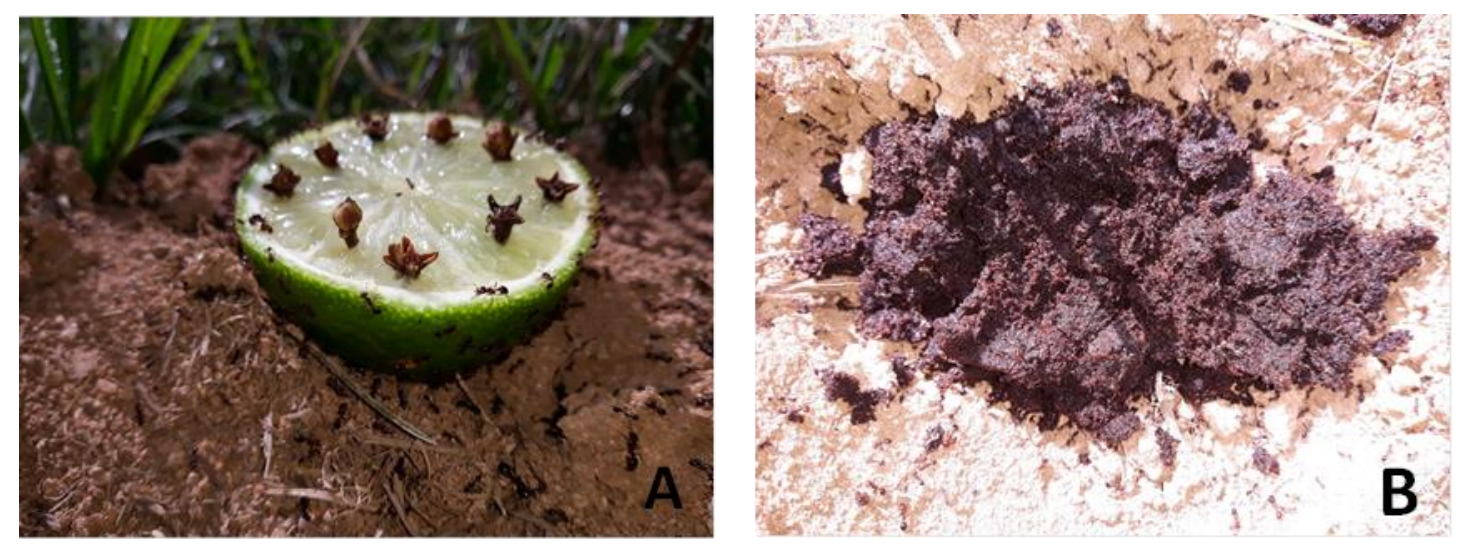

Figura 1. Teste do uso de Limão com cravo da Índia (A) e borra de café (B) no combate às formigas lava-pés.

O controle biológico foi recomendado em apenas duas publicações, uma em 2007 e outra em 2014 (Tabela 1). Esse método consiste na utilização de inimigos naturais como predadores (galinhas, rãs e sapos), parasitoides (moscas forídeos) e organismos entomopatogênicos (fungos e nematoides) para controlar as pragas. Por ser um procedimento realizado em laboratórios de pesquisa e que exige amplo conhecimento sobre as espécies envolvidas, não está acessível para a população, o que inviabiliza a sua prática.

Em 44,74\% das publicações $(n=17)$ foi recomendado o uso de controle químico (Tabela 1). O uso indiscriminado de produtos químicos para combater formigas lava-pés é motivo de preocupação, pois, a maioria dos produtos utilizados (biopesticidas, fipronil, iscas tóxicas e formicidas) exigem equipamentos de segurança para manipulação e aplicação (como luvas e máscaras), além do cuidado em não contaminar o meio ambiente e/ou outros animais que vivem naquele local. Na grande maioria dos vídeos e sites encontrados, pode-se notar que essas medidas de segurança não foram adotadas e nem esclarecidas à população. Vale ressaltar que estes produtos quando inalados ou em contato com a pele, podem levar a uma série de sintomas como desmaios e queimaduras, sendo que em casos mais graves, pode levar o paciente à morte por intoxicação aguda.

A primeira publicação encontrada data de 1997, seguida por uma segunda em 2004. A inconstância em publicações sobre o controle de lava-pés permanece até 2009 e, a partir de 2011, as publicações se tornam regulares (Figura 2). Caracteristicamente as produções bibliográficas são mais antigas e estão 
concentradas entre os anos de 1997 a 2011. A partir de 2007 se constata o aparecimento de informações de controle em sites que, embora irregulares, aparecem também nos últimos anos (2018 e 2019). Curiosamente os vídeos do YouTube, apesar de aparecerem em 2009, são mais frequentes a partir de 2014. Cabe destacar que as informações sobre a data de publicação só foram encontradas para 76,3\% (n = 29) do total registrado, o que dificulta em parte uma análise mais apurada sobre a cronologia das publicações.

A concentração de publicações sobre controle de lava-pés em sites e vídeos principalmente nos últimos 7 anos (2012 a 2019) (Figura 2), pode ser explicada pelo aumento da acessibilidade dos brasileiros à internet, por meio de notebook e celulares. As publicações veiculadas nessas mídias são um fenômeno atual na busca por qualquer tipo de informação. A facilidade para se publicar conteúdos na internet e nas plataformas de vídeo como o YouTube também contribuem para o aumento dessas publicações. Atualmente qualquer pessoa pode facilmente fazer uma filmagem e postar imediatamente em uma plataforma de vídeo (Tech Tudo, 2015).

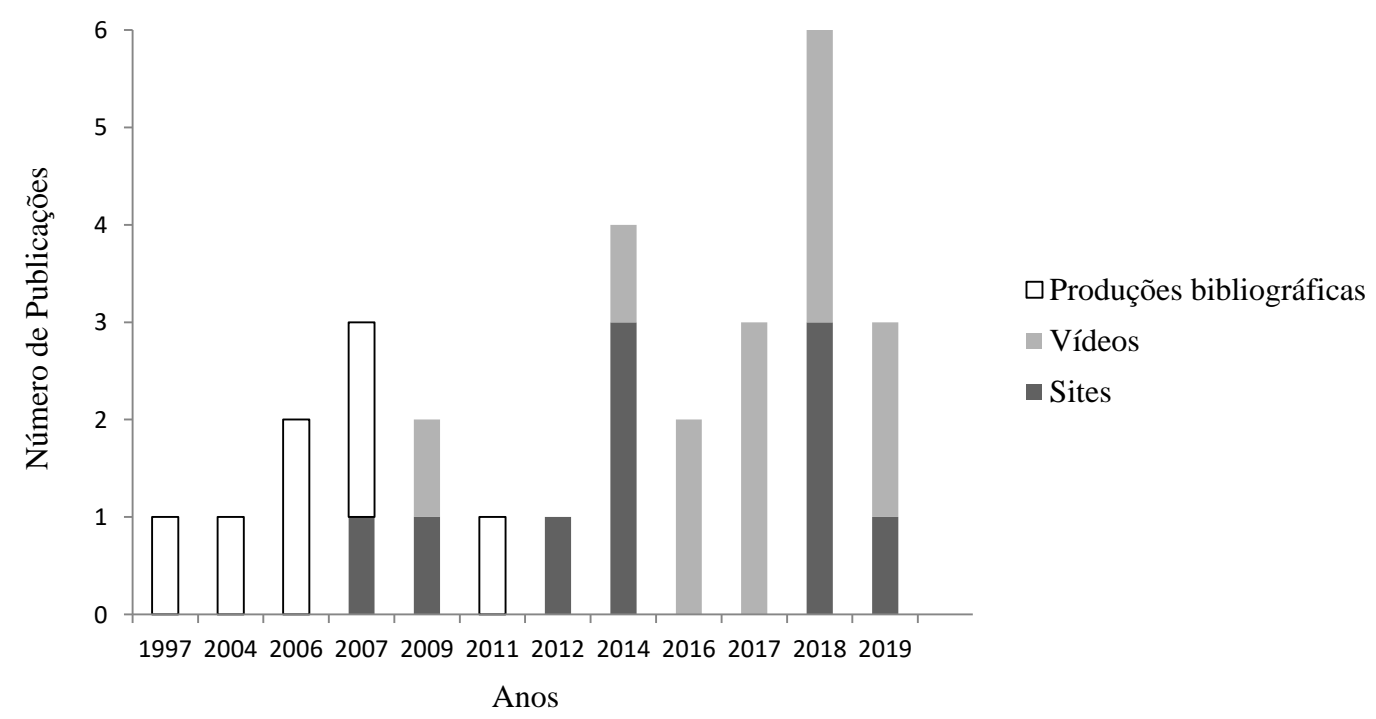

Figura 2. Distribuição das publicações sobre o controle de formigas lava-pés no Brasil (produções bibliográficas, vídeos e sites) nos últimos 22 anos.

Outro aspecto interessante revelado foi que em apenas 23 publicações (61\%) foi possível se detectar a presença/participação de um especialista (profissional e ou pesquisador) junto à recomendação de controle. Esse dado é preocupante, pois demonstra que em uma parcela significativa (cerca de $40 \%$ ) as recomendações de controle de formigas lava-pés são feitas sem a consultoria de um profissional qualificado, o que pode como consequência acarretar a proliferação de recomendações de controle sem qualquer qualidade comprovada. Soma-se a este fato uma importante particularidade associada às consultas realizadas na internet, que é a pronta aceitação por parte do espectador que rapidamente absorve aquele conteúdo e o toma como a melhor solução para o seu problema sem questioná-lo. 


\section{CONCLUSÃO}

Nossa pesquisa revelou que muitas das informações veiculadas não possuem fundamentação científica e podem colocar a pessoa que venha a realizá-la em sério risco durante a manipulação e aplicação do produto. É de grande importância que a população verifique a procedência das informações veiculadas em sites e vídeos, o que pode evitar acidentes durante a manipulação do produto, bem como garantir uma maior eficácia do método de controle aplicado.

\section{REFERÊNCIAS BIBLIOGRÁFICAS}

Botkin, D. \& Keller, E. 2011. Ciência ambiental: Terra, um Planeta Vivo. Rio de Janeiro, LTC. 681p.

Bueno, O.C. \& Bueno, F.C.B. 2007. Controle de formigas em áreas urbanas, pp.67-78. In: Pinto, A.S.; Rossi, M.M. \& Salmeron, E. (org.). Manejo de Pragas Urbanas. Piracicaba, CP 2. 208p.

Bueno, O.C.; Campos, A.E.; Morini, M.S. 2017. Formigas em Ambientes Urbanos no Brasil. Bauru, Canal 6 editora. 690p.

Campos-Farinha, A.E.C.; Júnior, J.J.; Bergman, E.C.; Zorzenon, F.J. \& Netto, S.M.R. 1997. Formigas Urbanas. Boletim Técnico do Instituto. Biólogico 8: 5-20.

Campos-Farinha, A.E.C.; Bueno, O.C.; Campos, M.C.G. \& Kato, L.M. 2002. As formigas urbanas no Brasil: retrospecto. Biológico 64: 129-133.

Campos-Farinha, A.E.C. \& Bueno, O.C. 2004. Formigas urbanas: comportamento e controle. Biológico 66: 47-48.

Creighton, W.S. 1930. The New World Species of the Genus Solenopsis (Hymenop. Formicidae). Proceedings of the American Academy of Arts and Sciences 66: 39-152.

Cumberland, M. \& Kirkman, K. 2012. The effects of disturbance on the red imported fire ant (Solenopsis invicta) and the native ant community. Forest Ecology and Management 279: 27-33.

Fernandes, E.F.; Santos-Prezoto, H.H.; Prezoto, F. 2016. Formigas lava-pés em ambientes urbanos: Bioecologia e risco de acidentes. CES Revista 30: 25-42.

Magro, S.R.; Ribeiro, L.J.R. \& Florim, A.C.P. 2007. Controle de pragas em residências. pp. 163-171. In: Pinto, 
A.S.; Rossi, M.M. \& Salmeron, E. (org.). Manejo de Pragas Urbanas. Piracicaba, CP 2, 208p.

Pereira, W.H. 2012. Práticas alternativas para a produção agropecuária agroecologia. Disponível em: https://ciorganicos.com.br/biblioteca/praticas-alternativas-para-a-producao-agropecuariaagroecologia/. Acesso em: 17 jun. 2019.

Souto, R.N.P.; Harada, A.Y. \& Maia, J.G.S. 2011. Estudos preliminares da atividade inseticida de óleos essenciais de espécies de Piper linneus (Piperaceae) em operárias de Solenopsis saevissima f Smith (Hymenoptera: Formicidae), em laboratório. Biota Amazônia 1: 42-28.

Tech Tudo. 2015. YouTube faz 10 Anos: Descubra Qual Foi o Primeiro Vídeo do Site. Disponível em: <https:// www.techtudo.com.br/noticias/noticia/2015/02/youtube-faz-10-anos-descubra-qual-foi-oprimeiro-video-do-site.html>. Acesso em: 17 jun. 2019.

Tschinkel, W.R. 2006. The Fire Ants. Cambridge, Harvard University Press, The Belknap Press. 752p.

Vinson, B. \& Sorensen, A. 1986. Imported Fire Ants, Life History and Impact. Austin, Texas Department of Agriculture. 28p.

Zarzuela, M.F.M. 2007. Utilização de Entomopatógenos para o Controle de Formigas Lava-pés. In: XVIII Simpósio de Mirmecologia, São Paulo, Brasil.

Zeringóta, V.R.; Castro, M.M.; Della Lucia, T.M.C.; Prezoto, F. 2014. Nesting of the fire ant Solenopsis saevissima (Hymenoptera: Formicidae) in an urban enviroment. Florida Entomologist 97: 668-673.

Zorzenon, F.J \& Campos-Farinha, A.E. de C. 2006. Principais pragas em gramados: biologia e controle (cupins, formigas cortadeiras e formigas lava-pés). In: III Simpósio Sobre Gramados, 2006, Botucatu, Brasil. 11p.

Zuben, A.P.B.; Almeida, M.G.R.; Lira, E.S. 2006. Manual de Controle Integrado de Pragas. Disponível em: http://www.campinas.sp.gov.br/sa/impressos/adm/FO086.pdf. Acesso em: 15 jun. 2019. 